\title{
Non-ideal multifrequency cloaking using strongly dispersive materials
}

\author{
A.E. Serebryannikov*, Ekmel Ozbay \\ Nanotechnology Research Center-NANOTAM, Department of Physics, Department of Electrical Engineering, Bilkent University, 06800 Ankara, Turkey
}

\section{A R T I C L E I N F O}

Keywords:

Cloaking

Fabry-Perot resonator

Drude-Lorentz material

\begin{abstract}
A B S T R A C T
Non-ideal multifrequency cloaking of dielectric cylinders is expected to be realizable by using singlelayer coating shells, which are made of isotropic homogeneous dispersive materials. In the present paper, scattering on the coated cylinders is studied for the Drude-Lorentz materials with frequency dependent permittivity and permeability. The exploited physical mechanism is based on the FabryPerot-type radial resonances arising in the coating shells. The obtained simulation results confirm the possibility of a strong reduction of the total scattering cross section at several frequencies simultaneously. Resonance frequencies can be estimated by using simple algebraic equations. The suggested mechanism can be used for TE and TM polarizations within a wide range of the variation of the diameter-to-wavelength ratio.
\end{abstract}

(c) 2010 Elsevier B.V. All rights reserved.

\section{Introduction}

The cloaks that make the dielectric and conducting objects completely, or nearly completely, invisible have been in focus in turn extensively raising interest throughout the past three years. Several approaches have been suggested, which include those based on the use of transformational optics [1-5], non-resonant plasmonic (meta)material shells [6-9], transmission-line networks [10,11], and plasmonic resonance [12-14]. The first approach is considered as the most universal, in which the cloaking is realized due to the proper re-routing of electromagnetic waves around the object to be cloaked. The specially designed inhomogeneous metamaterials with anisotropy need to be used, while for cylindrical objects magnetism is required only if the electric field is polarized along the axis. It was demonstrated that the coordinate variations of the tensor components that are required for this approach can be obtained in one- and two-dimensional layered structures with isotropic constituents, some of which can be associated with circular photonic crystals [15-18].

The second approach is based on the negative local polarizability of plasmonic materials and metamaterials, of which the cloaking shell is made. In this case, the scattering processes from the shell and the dielectric object to be cloaked might compensate for each other. Both of the mentioned approaches enable cloaking in a wide range of the object-size-to-wavelength ratio, including

\footnotetext{
* Corresponding author.

E-mail addresses: aeserebr@gmail.com (A.E. Serebryannikov), ozbay@bilkent.edu.tr (E. Ozbay).
}

the values corresponding to resonant-sized objects. The third and fourth approaches are based on the travelling of electromagnetic waves through the cloak and on the localized plasmonic resonance, respectively. Special attention has been paid to the possibility of achieving a broadband and multifrequency cloaking $[5,11,17,19-22]$. In particular, it has been shown that it is sufficient to use a two-layer coating, which is made of isotropic homogeneous plasmonic materials with properly chosen parameters, in order to cloak a spherical particle at two frequencies simultaneously [21]. The possibility of using the frequency dispersion of plasmonic materials for realizing cloaking at several frequencies in multilayered structures has been discussed in [22].

The main goal of the present paper is to demonstrate the potential of an alternative approach for obtaining a substantial reduction of the total scattering cross section, i.e., non-ideal cloaking, at multiple non-equidistant frequencies. It is based on the coating of the objects to be cloaked with the shells that are made of isotropic inhomogeneous dispersive materials. The suggested heuristic approach is based on the assumption that multiple Fabry-Perot-type radial resonances might appear in cylindrical structures made of high-index materials. In turn, a high frequency-dependent index of refraction can be obtained by using Drude-Lorentz artificial materials, as well as materials that are characterized by the dispersion of another type. We will show that in case of an empty coating shell, which is made of a nondispersive high-index material, multiple minima of the total scattering cross section do appear, in turn corresponding to the frequencies of total transmission in case of a planar Fabry-Perot resonator with the same thickness and material parameters. This correspondence remains for dispersive materials, for which Fabry-Perot resonance frequencies are non-equidistant, while 
placing a dielectric cylinder inside a shell results in a relatively slight shift of the frequencies. Extensive numerical simulations were carried out in order to validate the suggested approach.

\section{Theoretical background}

We studied the scattering of electromagnetic plane waves on a dielectric cylinder of radius $r$ that was coated with a ring shell with the inner and outer radii $r$ and $R$, which is made of a material showing Drude-Lorentz dispersion. The permittivity and permeability of the core and those of the shell are denoted by $\varepsilon_{c}, \mu_{c}, \varepsilon_{s}$, and $\mu_{s}$, respectively. According to [23], $\varepsilon_{s}$ and $\mu_{s}$ are given by

$\varepsilon_{s}=1-C \omega_{p e}^{2} /\left(\omega^{2}-i \Gamma_{e} \omega-\omega_{0 e}^{2}\right)$

and

$\mu_{s}=1-G \omega_{p m}^{2} /\left(\omega^{2}-i \Gamma_{m} \omega-\omega_{0 m}^{2}\right)$

where $\omega$ is angular frequency. If $\omega_{0 e}=\omega_{0 m}, \omega_{p e}=\omega_{p m}, \Gamma_{e}=\Gamma_{m}$, and $C=G$, the shell is matched to the outer air space at all frequencies.

The normalized total scattering cross section is calculated as

$\sigma=(k R)^{-1} \sum_{n=-\infty}^{\infty} a_{n}^{2}$

where $a_{n}$ is the $n$ th-order diffraction amplitude, which is given by the analytical formula containing some combinations of the cylindrical functions, which is obtained by using the FourierBessel series representations of the field and conservation of the tangential components at the boundaries, and $k=\omega / c$ is the freespace wavenumber. Both TE ( $H$-field is parallel to the cylinder axis) and TM (E-field is parallel to the cylinder axis) polarizations of the incident wave will be considered by placing an emphasis on those cases, wherein a strong reduction of $\sigma$ can be obtained due to the coating.

In line with the theory of planar Fabry-Perot resonators, the dependence of transmittance $\tau$ on $\omega$ and the angle of incidence $\theta$ is given by the well-known Airy formula:

$\tau=(1-\rho)^{2} /\left[(1-\rho)^{2}+4 \rho \sin ^{2}(n k D \cos \theta)\right]$

where $\rho, D$, and $n$ stand for mirror reflectance, distance between the mirrors, and the index of refraction of the filling medium, respectively. It follows from (4) that $\tau=1$ if

$n k D \cos \theta=\pi m$,

where $m=0, \pm 1, \pm 2, \ldots$. The total transmission occurring in such a resonator means that a far-zone observer located in the incidence/reflection half-space does not see it at multiple equidistant frequencies. The basic idea of the studied cloaking mechanism originates from the analogy between the totaltransmission regime in the planar Fabry-Perot resonator and the vanishing total scattering cross section in the corresponding ring shell. In other words, the wave is expected to go through the cylindrical shell without scattering in a similar way as in the transmission-line networks approach [10]. Correspondingly, wave processes at the front and back side interfaces in the planar case, and at the outer and inner interfaces in the cylindrical case, are analogous so that (5) might be used in the latter case, provided that $r \gg \lambda_{s}$ where $\lambda_{s}$ is wavelength in the shell material, i.e., $n$ is rather large. If $\tau=1$ in the planar case, a far-field observer should not see the corresponding cylindrical structure.

Firstly, we consider a simplified case, when $\varepsilon_{s}$ and $\mu_{s}$ do not depend on $\omega$ and the shell is empty, i.e. $\varepsilon_{c}=\mu_{c}=1$. Fig. 1 shows $\sigma$ vs $k D$ in this case. One can clearly see the multiple equidistant minima, at which $\sigma$ tends to vanish. Their locations are in good agreement with the $k D$ values obtained from (5) where $n$ is

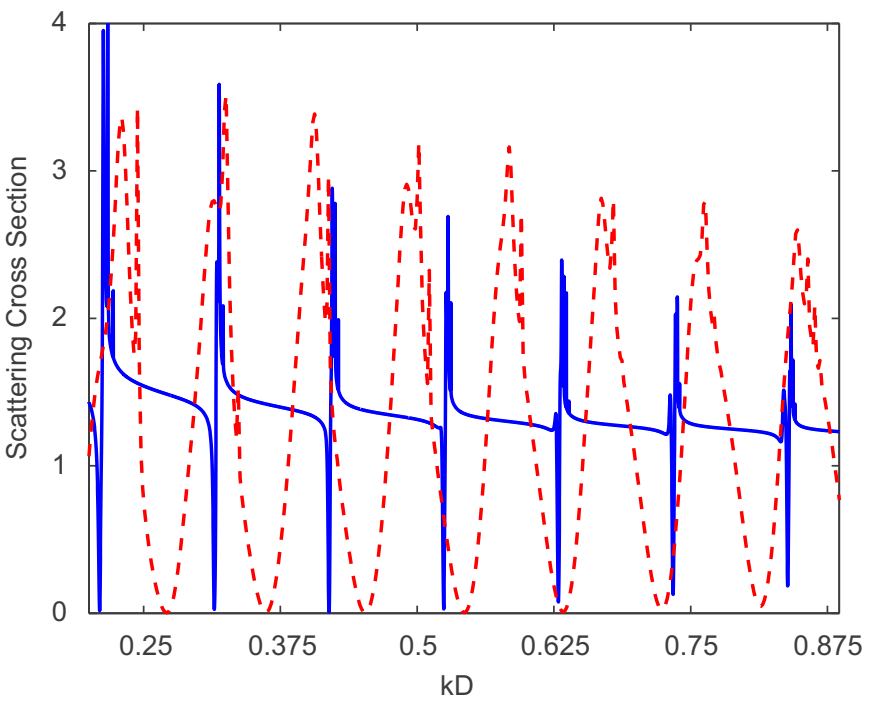

Fig. 1. Total scattering cross section vs $k D$ at $\varepsilon_{s}=900$ and $\mu_{s}=1$ (blue solid line), and $\varepsilon_{s}=\mu_{s}=35.4$ (red dashed line); $R / r=1.4, \varepsilon_{c}=\mu_{c}=1$, TM polarization. (For interpretation of the references to color in this figure legend, the reader is referred to the web version of this article.)

Table 1

Comparison of the $k D$ values for the minima of $\sigma$ in the empty shells in Fig. 1 (second and fourth lines), and for $\tau=1$ in the corresponding planar resonators (third and fifth lines).

\begin{tabular}{llllll}
\hline$m$ & 2 & 3 & 4 & 5 & 6 \\
\hline Blue line & 0.2098 & 0.3147 & 0.4196 & 0.5243 & 0.6291 \\
Eq. (5) & 0.2094 & 0.3142 & 0.4189 & 0.5236 & 0.6283 \\
Red line & 0.1811 & 0.2714 & 0.3619 & 0.4524 & 0.5429 \\
Eq. (5) & 0.1775 & 0.2662 & 0.355 & 0.4437 & 0.5325 \\
\hline
\end{tabular}

replaced by $n_{s}=\sqrt{\varepsilon_{s}} \sqrt{\mu_{s}}$ and $\theta=0$. Hence, the shell itself becomes nearly invisible when the $k D$ value corresponds to the totaltransmission regime of the planar Fabry-Perot resonator. The $k D$ values taken from Fig. 1 and those calculated by using (5) at $\theta=0$ are shown in Table 1 for TM polarization. A similar result was obtained for TE polarization. Thus, our assumption concerning the analogy between high-index planar and cylindrical (ring) structures is quite justified. From the comparison of the cases with $\mu_{s}=1$ and $\mu_{s} \neq 1$ in Fig. 1, it follows that the presence of magnetism enables the obtaining of much wider resonances. This feature is connected, in fact, with the dependence of $\rho$ on the impedance of the filling medium $Z_{s}=\sqrt{\mu_{s} / \varepsilon_{s}}$.

Since the analogy with the planar Fabry-Perot resonators works well in a dispersionless case, it is easily expecting that it also works in the case of dispersive material. Before analyzing the far- and near-field effects for dielectric cylinders coated with such shells, we consider the general features of the location of the total-transmission frequencies for planar Fabry-Perot resonators filled with a Drude-Lorentz material. To do this, we substitute $\varepsilon_{c}$ and $\mu_{c}$ given by (1) and (2) to (5), assuming $C=G=1, \theta=0$, and $n=n_{c}$, and neglecting by $\operatorname{Im} \varepsilon_{c}$ and $\operatorname{Im} \mu_{c}$. In the impedance-matched case, $\varepsilon_{c}=\mu_{c}$, we obtain the following equation:

$K^{3}-\Lambda_{m} K^{2}-\left(K_{p}^{2}+K_{0}^{2}\right) K+\Lambda_{m} K_{0}^{2}=0$,

where $K=\omega D / c, \quad K_{p}=k_{p} D, \quad K_{0}=k_{0} D, \quad k_{p}=\omega_{p e} / c=\omega_{p m} / c$, $k_{0}=\omega_{0 e} / c=\omega_{0 m} / c$, and $\Lambda_{m}=\pi m$. For a purely dielectric DrudeLorentz medium $\left(\mu_{c}=1\right)$, this approach leads to

$K^{4}-\left(K_{p}^{2}+K_{0}^{2}+\Lambda_{m}^{2}\right) K^{2}+\Lambda_{m}^{2} K_{0}^{2}=0$ 
a

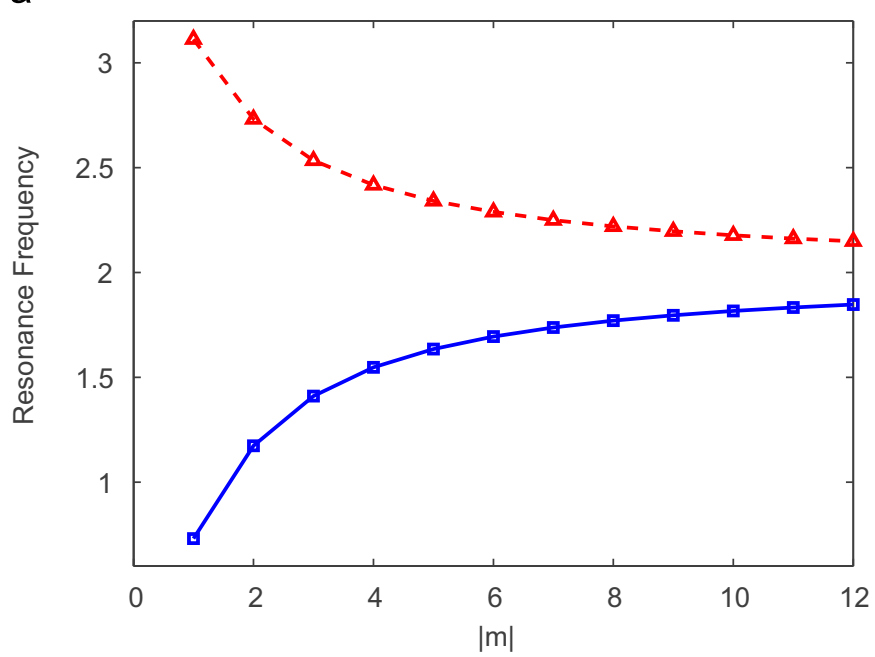

b

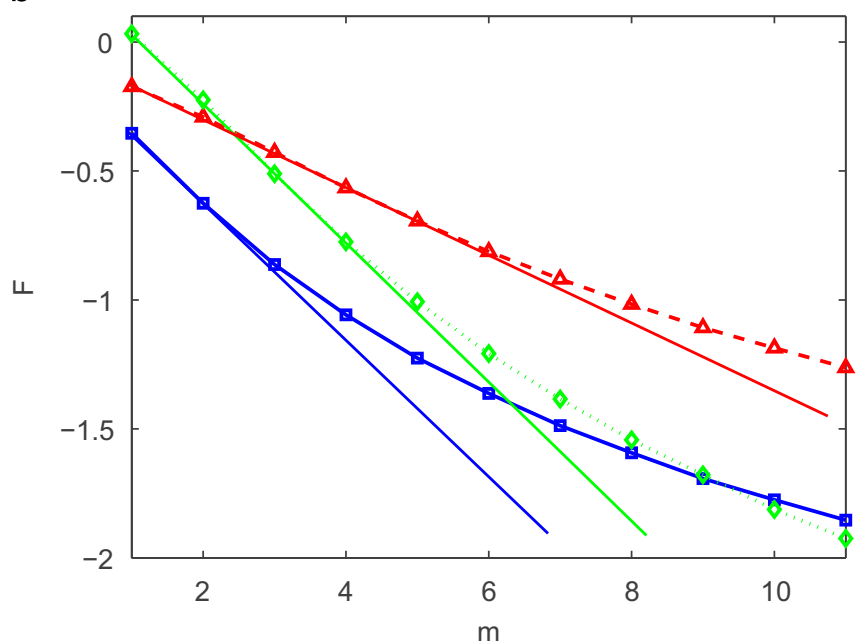

Fig. 2. Normalized resonance frequency of the first (blue squares) and second (red triangles) groups in units of $k D=k(R-r)$ vs $|m|$ at $\omega_{p e} D / c=\omega_{p m} D / c=4$ and $\omega_{0 e} D /$ $c=\omega_{0 m} D / c=2$-plot (a); Increment of the resonance frequency of the first group at the same parameters as in plot (a) (blue squares), at $\omega_{p e} D / c=\omega_{p m} D / c=8$ and $\omega_{0 e} D$ $c=\omega_{0 m} D / c=4$ (red triangles), and at $\omega_{p e} D / c=8, \omega_{0 e} D / c=4$, and $\omega_{p m} D / c=0$ (green rhombs)—plot (b); $R / r=1.4, \Gamma_{e} / \omega_{p e}=\Gamma_{m} / \omega_{p m}=10^{-6}, C=G=1$. (For interpretation of the references to color in this figure legend, the reader is referred to the web version of this article.)

where $K_{p}=k_{p} D=\omega_{p e} D / c$ and $K_{0}=k_{0} D=\omega_{0 e} D / c$. The resonance frequencies are found by solving (6) and (7) for $K$.

Fig. 2(a) shows an example of the location of the first and second roots of (6) as a function of $m$. The small non-zero values of $\Gamma_{e}$ and $\Gamma_{m}$ were used in the calculations. The basic feature observed is that the resonance frequencies are located more densely at increasing $m$, i.e., at decreasing $\left|\omega-\omega_{0 e}\right|$. The first group of the roots corresponds to the positive values of $m, \varepsilon_{s}$ and $n_{s}$, and the second one corresponds to the negative values of $m, \varepsilon_{s}$ and $n_{s}$. A proper choice of dispersion should provide one with a required number of the resonances within a fixed frequency range.

Note that the dense eigenmode spectra have been found in some non-linear problems of laser optics and plasma physics $[24,25]$. Although the densening is connected in our case with a quite different physical mechanism, it would be interesting to check as to whether the roots of (6) can show some kind of selfsimilarity. To do this, we plot the $m$-dependence of the logarithmic increment $F(m)=\lg A(m)$, where $A(m)=\hat{K}_{m+1}-\hat{K}_{m}$, and $\hat{K}_{m+1}$ and $\hat{K}_{m}$ are the roots of the first group, see Fig. 2(b). The constant slope occurs for several first $m$, in turn leading to a finite scaling interval. It is seen that both the slope and interval width depend on the problem parameters. Within the scaling intervals, the increments approximately satisfy the equation $A(m)=B 10^{-b m}$, where $b$ and $B$ are the constants depending on the problem parameters which include $\hat{K}_{1}$. Hence, the dependence of $n_{s}$ on $\omega$ can itself be a cause of the appearance of self-similarity. The same occurs for the second group of the roots, which corresponds to $n_{s}<0$. Similar features in the location of $\hat{K}_{m}$ are observed for the first group of the roots of (7), i.e. at $\mu_{s}=1$. However, in this case there is no analog of the second group from Fig. 2(a), since $n_{s}$ is imaginary-valued at $\omega_{0 e}^{2}<\omega^{2}<\omega_{p e}^{2}+\omega_{0 e}^{2}$.

\section{Results and discussion}

Let us check as to whether the multiple minima of $\sigma$ appear for a shell made of a dispersive material, when a dielectric cylinder is

a

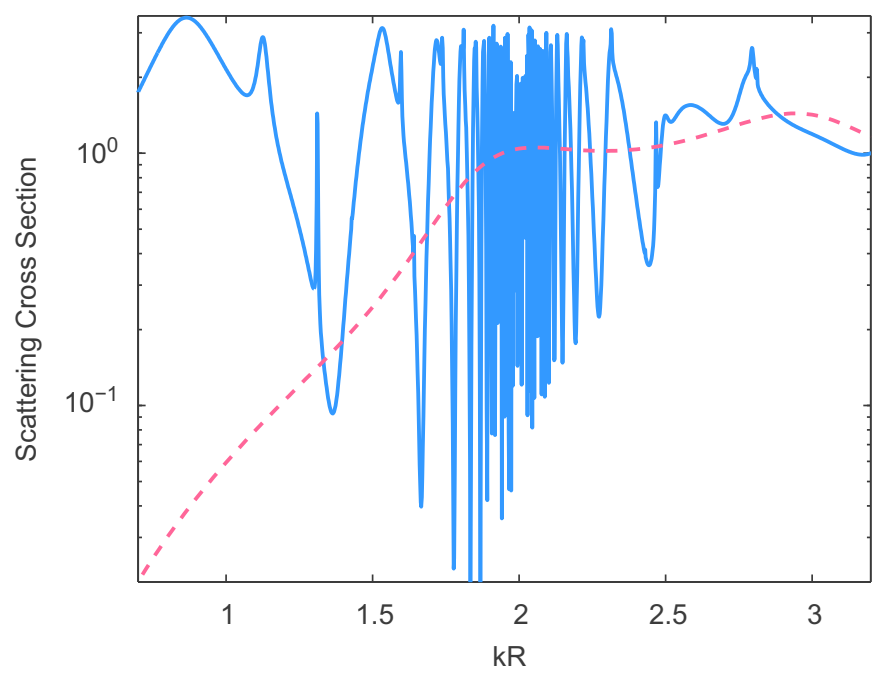

b

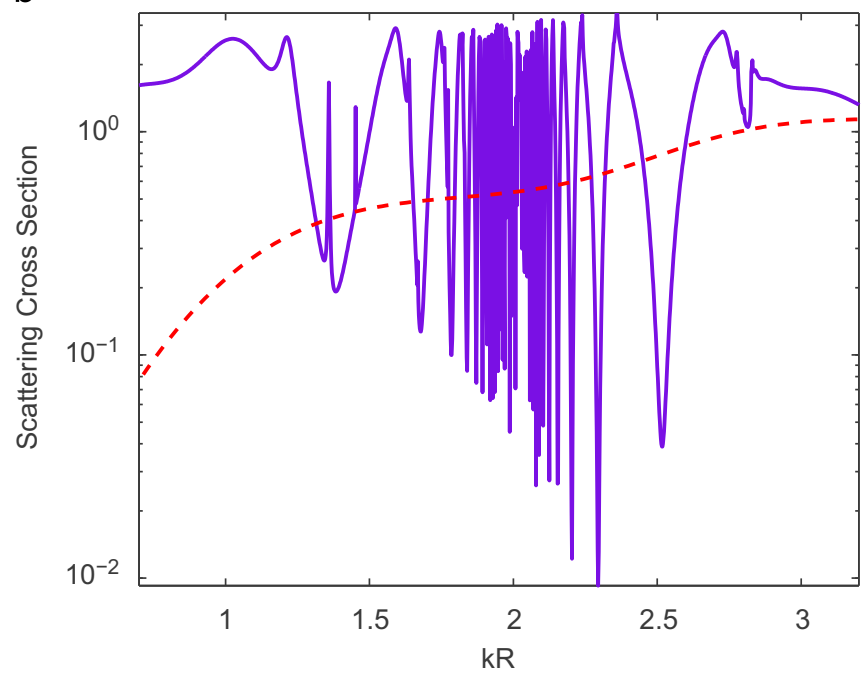

Fig. 3. Total scattering cross section vs $k R$ at $\omega_{p e} R / c=\omega_{p m} R / c=2.83, \omega_{0 e} R / c=\omega_{0 m} R /$ $c=2$ and $\Gamma_{e} / \omega_{p e}=\Gamma_{m} / \omega_{p m}=10^{-6}$ (blue solid line), $\varepsilon_{s}=\mu_{s}=1$ (pink dashed line), $\varepsilon_{c}=5.8, \mu_{c}=1$, TE polarization-plot (a); and at $\omega_{p e} R / c=\omega_{p m} R / c=2.83, \omega_{0 e} R /$ $c=\omega_{0 m} R / c=2$ and $\Gamma_{e} / \omega_{p e}=\Gamma_{m} / \omega_{p m}=10^{-6}$ (violet solid line), $\varepsilon_{s}=\mu_{s}=1$ (red dashed line), $\varepsilon_{c}=2.8, \mu_{c}=1$, TM polarization-plot (b); $R / r=2.0, C=G=1$. (For interpretation of the references to color in this figure legend, the reader is referred to the web version of this article.) 
located inside the shell. Fig. 3 shows $\sigma$ vs $k R$ for the two representative cases, in which the shells show the same impedance as free space. Losses were kept low in order to better see the possible manifestations of the Fabry-Perot-type resonances. The multiple minima of $\sigma$ were observed, which became denser while the difference between $k R$ and $\omega_{0 e} R / c$ was decreased. Among these minima, there are ones that are characterized by a more than tenfold reduction of $\sigma$. Typically, $\sigma$ at the minima takes the values from 0.02 to 0.04 , so that one can consider the observed reduction as the non-ideal cloaking. In Fig. 3, the minimal $\sigma \approx 0.01$ occurs in plot (b) at $k R=2.295$. In fact, the structures in Fig. 3 correspond to a planar Fabry-Perot resonator that is placed between two different media. However, because of a relatively large index contrast between the shell and core and a relatively small contrast between the core and free space, the latter only leads to a slight shift of a resonance frequency. In fact, it plays a role of perturbation, while the values of $\hat{K}_{m}$ can still be estimated using (6). Note that the range of $\sigma<0.1$ in case with the coating starts from $\varepsilon_{s}=\mu_{s} \approx 7.5$ and $m=2$ in the vicinity of $k R=1.67$ in Fig. $3(\mathrm{a})$, and from $\varepsilon_{s}=\mu_{s} \approx 14$ and $m=3$ in the vicinity of $k R=1.84$ in Fig. 3(b).

Both ranges with $\operatorname{Re} n_{s}>0 \quad\left(\omega<\omega_{0 e}\right)$ and $\operatorname{Re} n_{s}<0$ $\left(\omega_{0 e}<\omega<\sqrt{\omega_{p e}^{2}+\omega_{0 e}^{2}}\right)$ similarly contribute to the multifrequency reduction of $\sigma$. However, either the former as in Fig. 3(a), or the latter as in Fig. 3(b), enables the obtaining of the strongest reduction of $\sigma$ for a specific parameter choice. For the examples shown in Fig. 3, the multifrequency cloaking corresponds to $2 r / \lambda \approx 1 / 3$, where $\lambda$ is free-space wavelength. This ratio can be increased owing to a more careful parameter choice. For example, $2 r / \lambda=0.45$ at the same parameters as in Fig. 3(b), except for $R / r=1.4$, for both polarizations. At the minimum of $\sigma$ near $k R=2.52$ in Fig. $3(\mathrm{~b}), \varepsilon_{s} \approx-2.5$ and $m=-1$.

A typical example of the axial field distribution at a minimum of $\sigma$ is shown in Fig. 4. The resonant-type distribution with the dominant role of the zero space harmonic occurs inside the core. Hence, the incident-wave energy can be concentrated due to the inner resonance. This feature distinguishes the suggested approach from the most known cloaking approaches. In turn, the field in the shell shows the multiple alternating minima and maxima in the radial direction and rather weak dependence on the azimuthal coordinate. This is consistent with the theory of

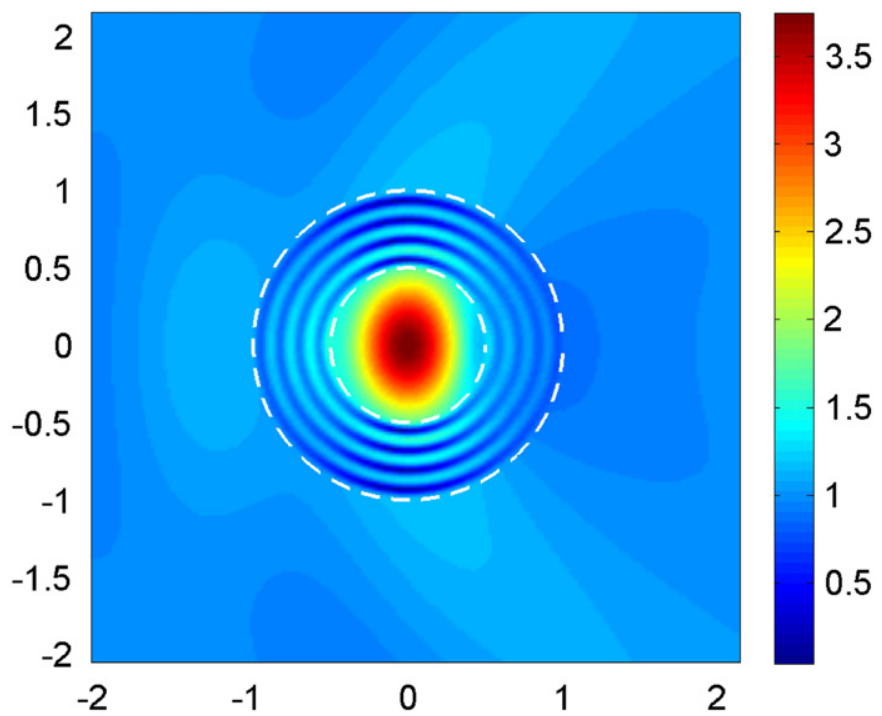

Fig. 4. Modulus of the axial field at $k R=1.834$ and the same remaining parameters as in Fig. 3(a); the unity amplitude equals to that of the incident wave; the structure is illuminated from the side of the negative abscissa values.

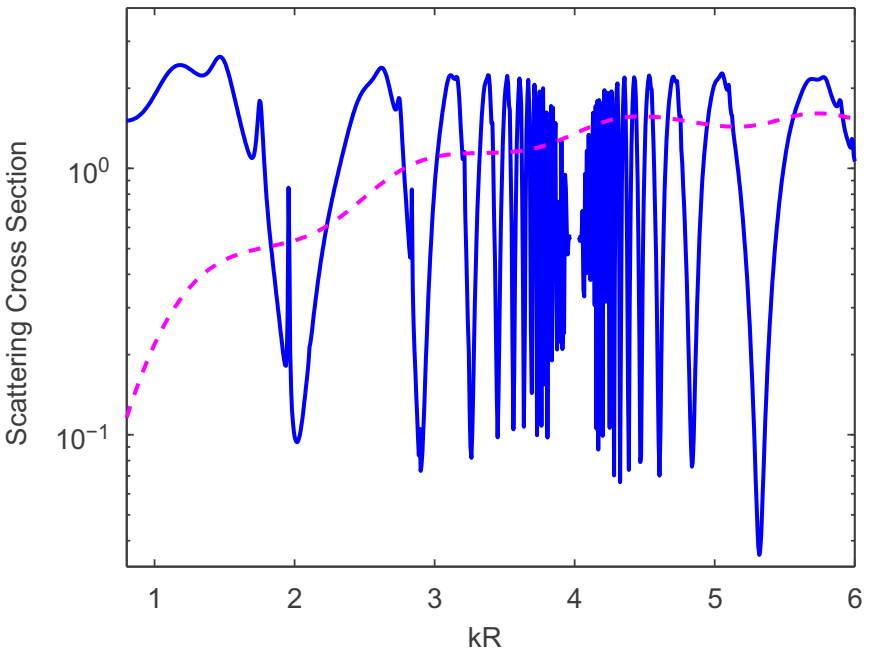

Fig. 5. Total scattering cross section $\omega_{p e} R / c=\omega_{p m} R / c=6.76, \omega_{0 e} R / c=\omega_{0 m} R / c=4$, $\Gamma_{e} / \omega_{p e}=\Gamma_{m} / \omega_{p m}=10^{-4}, C=G=1$ (dark blue solid line), and $\varepsilon_{s}=\mu_{s}=1$ (pink dashed line); $\varepsilon_{c}=2.8, \mu_{c}=1, R / r=1.4$, TM polarization. (For interpretation of the references to color in this figure legend, the reader is referred to the web version of this article.)

planar Fabry-Perot resonators. In fact, the wave propagating in the radial direction within the shell weakly sees the curvature, in turn producing a weak radial field component. The observed nearfield features are typical for the resonances with large $m$ and small $\sigma$. Here, $m=4$ and $\sigma \approx 0.017$. This example shows that a volumetric mode excited inside the shell can co-exist with a regime of weak scattering. Note that in our case this effect was obtained in an azimuthally uniform structure. The illustrative phase maps for purely dielectric high-index shells can be found in [26]. The weaker the scattering, the weaker the phase modification is outside of the shell.

Fig. 5 demonstrates that not only subwavelength objects, but also resonant-sized objects can be cloaked, at least at relatively small $\varepsilon_{c}$. The increase of $\omega_{0 e}, \omega_{0 m}, \omega_{p e}$, and $\omega_{p m}$, and decrease of $R / r$ are natural ways to obtain multifrequency reduction at $2 r / \lambda \geq 1$. However, the parameters should be carefully adjusted. In particular, the use of the negative-index band allows for increasing $2 r / \lambda$. For example, a more than tenfold reduction of $\sigma$ is achieved at $k R>\omega_{0 e} R / c$, while $2 r / \lambda$ is varied from 0.94 to 1.21 . The range of $\sigma<0.1$ extends here from 2.02 to 5.32, and involve the resonances with $|m| \geq 1$. Those with $m=1$ and $m=-1$ correspond to $k R=2.02$ and $\operatorname{Re} \varepsilon_{s}=4.835$, and $k R=5.32$ and $\operatorname{Re} \varepsilon_{s}=-2.715$, respectively. Owing to a rather large $\omega_{0 e} R / c$, the minima are significantly shifted here with respect to those obtained using (5), despite of a small index contrast between the core and shell.

Until now, we considered the cases of low losses. However, the losses can set a strong restriction on the applicability of the suggested approach due to suppressing the resonances at large $|m|$ and limiting the range of the variation of $\operatorname{Re} \varepsilon_{s}$ and $\operatorname{Re} \mu_{s}$. A reasonable compromise between the losses, achievable range of variation of $\operatorname{Re} \varepsilon_{s}$ and $\operatorname{Re} \mu_{s}$, and the thickness of the shell is a basic engineering problem to be solved. Fig. 6 shows $\sigma$ vs $k R$ for the two cases with $\Gamma_{e}$ and $\Gamma_{m}$ being larger than in Figs. 3-5. Despite that the expected effects of the losses increase take place, the minima with near-zero $\sigma$ still exist, leading to the multifold weakening of the scattering as compared to the corresponding non-coated shells. In the case with $\varepsilon_{c}=2.8$ and $\Gamma_{e} / \omega_{p e}=10^{-3}$, several minima appear, for which $\sigma<0.04$. In the case with $\varepsilon_{c}=5.8$ and $\Gamma_{e} / \omega_{p e}=10^{-2}$, such a sole minimum has only been observed. The possibility of obtaining the deep minima of $\sigma$ at 


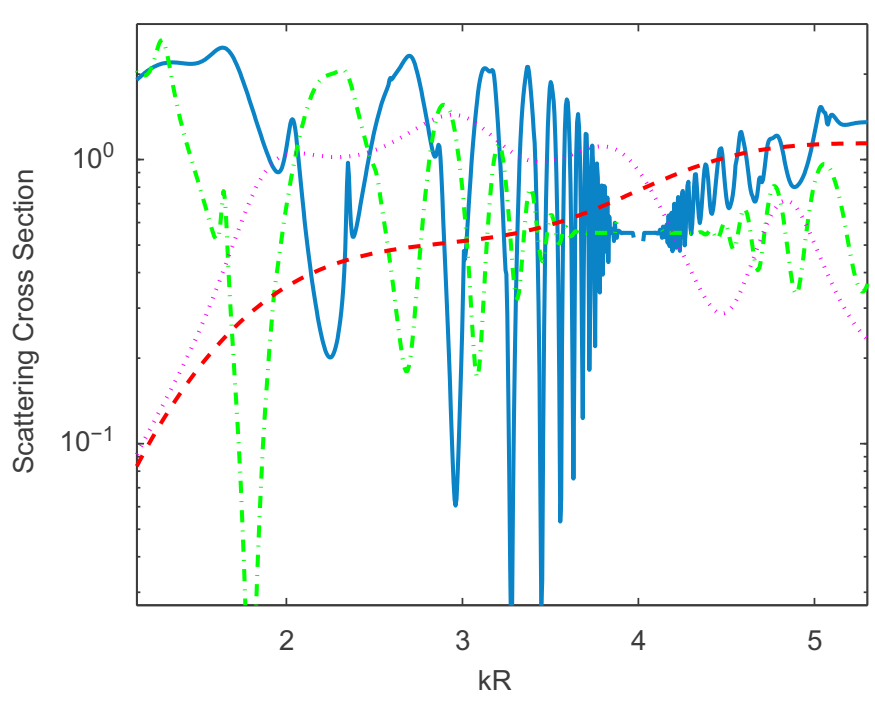

Fig. 6. Total scattering cross section at $\omega_{p e} R / c=\omega_{p m} R / c=4.47, \omega_{0 e} R / c=\omega_{0 m} R / c=4$, $\Gamma_{e} / \omega_{p e}=\Gamma_{m} / \omega_{p m}=10^{-3}, C=G=1, \varepsilon_{c}=2.8, \mu_{c}=1, R / r=3.2$, TM polarization-blue solid line; $\omega_{p e} R / c=\omega_{p m} R / c=5.66, \omega_{0 e} R / c=\omega_{0 m} R / c=4, \quad \Gamma_{e} / \omega_{p e}=\Gamma_{m} / \omega_{p m}=10^{-2}$, $C=G=1, \varepsilon_{c}=5.8, \mu_{c}=1, R / r=2$, TE polarization-green dash-dotted line; $\varepsilon_{s}=\mu_{s}=1$, $\varepsilon_{c}=2.8, \mu_{c}=1, R / r=3.2$, TM polarization-red dashed line; $\varepsilon_{s}=\mu_{s}=1, \varepsilon_{c}=5.8, \mu_{c}=1, R /$ $r=2$, TE polarization-pink dotted line. (For interpretation of the references to color in this figure legend, the reader is referred to the web version of this article.)

$\Gamma_{e} / \omega_{p e} \geq 10^{-2}$, at least for two $k R$-values simultaneously, is presently under study.

In the former case, $\sigma<0.1$ is achieved within the range that extends from $k R=2.96(m=2)$ to $3.63(m=6)$, corresponding to $\operatorname{Re} \varepsilon_{s}$ varied from 3.76 to 8.1 and $\operatorname{Im} \varepsilon_{s}$ varied from $-9 \times 10^{-3}$ to $-7.32 \times 10^{-2}$. For the minimum near $k R=3.63$, the figure of merit $(\mathrm{FOM})=\operatorname{Ren}_{s} /\left|\mathrm{Im} n_{s}\right| \approx 110$. In the latter case, the minimum of $\sigma$ at $k R=1.8$ corresponds to $m=1, \operatorname{Re} \varepsilon_{s}=3.51, \operatorname{Im} \varepsilon_{s}=-2.8 \times 10^{-2}$, and $\mathrm{FOM}=124$. According to Fig. 1, the multiple minima of $\sigma$ can appear for purely dielectric empty shells. The results obtained most recently show that the reduction of $\sigma$ can also be obtained without any magnetism, while placing a cylinder inside [26]. Fig. 7 shows that a significant reduction of $\sigma$ can be achieved at least at two $k R$-values simultaneously. Here $\operatorname{Re} \varepsilon_{c} \approx 33$, $\operatorname{Im} \varepsilon_{c}=-0.049$ and $m=1$, and $\operatorname{Re} \varepsilon_{c} \approx 123, \operatorname{Im} \varepsilon_{c}=-0.735$ and $m=2$ at the first and second deep minima, respectively. It is noteworthy that the results for the purely dielectric shells are still less promising from the point of view of multifrequency reduction of $\sigma$ than those for the metamaterial shells.

\section{Conclusions}

To summarize, we studied the scattering of plane electromagnetic waves on dielectric cylinders coated with the shells made of strongly dispersive materials. It was shown that the multiple minima of the total scattering cross section can appear, being connected with the resonances in the core and shell. In particular, the multiple Fabry-Perot-type radial resonances can appear in the shell. The non-ideal cloaking can be obtained simultaneously at several resonance frequencies, which nearly coincide with those of the total transmission in the corresponding planar Fabry-Perot resonators. While the Drude-Lorentz dispersion has been assumed for the shell materials, similar scattering features are expected to be obtainable for the dispersion of another type. A necessary condition is that the index of refraction of the shell material is varied within a wide range, providing the existence of Fabry-Perot-type resonances that are located rather close to each other. Engineering the cloaks that would involve

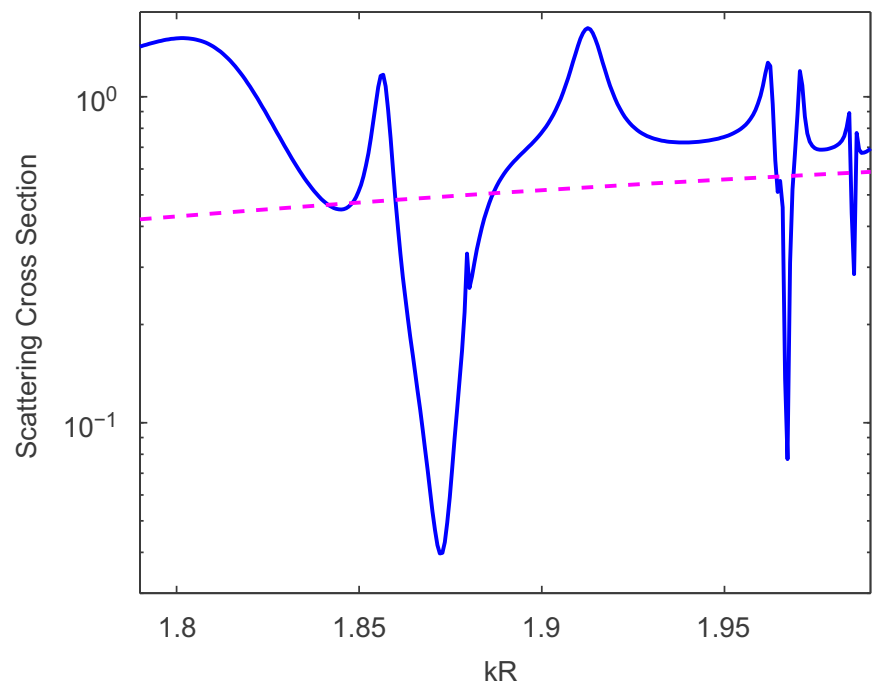

Fig. 7. Total scattering cross section at $\omega_{p e} R / c=4, \omega_{0 e} R / c=2$ and $\Gamma_{e} / \omega_{p e}=10^{-4}$ (dark blue solid line), and $\varepsilon_{s}=1$ (pink dashed line); $R / r=1.4, \varepsilon_{c}=2.8, \mu_{s}=\mu_{c}=1$, TE polarization. (For interpretation of the references to color in this figure legend, the reader is referred to the web version of this article.)

realistic lossy materials, and creation of a general theory of the suggested cloaking mechanism, will be in the focus of future studies.

\section{Acknowledgments}

This work is supported by the European Union under the projects EU.PHOME and EU.ECONAM, and TUBITAK under the project nos. 107A004 and 107A012. E.O. also acknowledges partial support from the Turkish Academy of Sciences.

\section{References}

[1] J.B. Pendry, D. Schurig, D.R. Smith, Science 312 (2006) 1780.

[2] D. Schurig, J.J. Mock, B.J. Justice, S.A. Cummer, J.B. Pendry, A.F. Starr, D.R. Smith, Science 314 (2007) 977.

[3] S.A. Cummer, B.-I. Popa, D. Schurig, D.R. Smith, J. Pendry, M. Rahm, A. Starr, Phys. Rev. Lett. 100 (2008) 024301.

[4] W. Cai, U.K. Chettiar, A.V. Kildishev, V.M. Shalaev, Opt. Express 16 (2008) 5444.

[5] A.V. Kildishev, W. Chai, U.K. Chettiar, V.M. Shalaev, New J. Phys. 10 (2008) 115029.

[6] M. Silveirinha, A. Alu, N. Engheta, Phys. Rev. E 75 (2007) 036603.

[7] A. Alu, N. Engheta, Opt. Express 15 (2007) 3318.

[8] A. Alu, N. Engheta, Phys. Rev. E 78 (2008) 045602.

[9] F. Bilotti, S. Tricarico, L. Vegni, New J. Phys. 10 (2008) 115035.

[10] P. Alitalo, O. Luukkonen, L. Jylhä, J. Venermo, S. Tretyakov, IEEE Trans. Ant. Propag. 56 (2008) 416.

[11] P. Alitalo, O. Luukkonen, J. Mosig, S. Tretyakov, Microwave Opt. Technol. Lett. 51 (2009) 1627.

[12] G.W. Milton, N.-A.P. Nicorovici, Proc. Roy. Soc. A 462 (2006) 3027.

[13] N.-A.P. Nicorovici, G.W. Milton, R.C. McPhedran, L.C. Botten, Opt. Express 15 (2007) 6314.

[14] N.-A.P. Nicorovici, R.C. McPhedran, S. Enoch, G. Tayeb, New J. Phys. 10 (2008) 115020.

[15] D. Xiao, H.T. Johnson, Opt. Lett. 33 (2008) 860.

[16] Y. Huang, Y. Feng, T. Jiang, Opt. Express 15 (2007) 11133.

[17] M. Farhat, S. Guenneau, A.B. Movchan, S. Enoch, Opt. Express 16 (2008) 5656.

[18] B. Kante, A. de Lustrac, J.M. Lourtioz, S.N. Burokur, Opt. Express 16 (2008) 9191.

[19] R. Liu, C. Ji, J.J. Mock, J.Y. Chin, T.J. Cui, D.R. Smith, Science 323 (2009) 366

[20] U. Leonhardt, T. Tyc, Science 323 (2009) 110.

[21] A. Alu, N. Engheta, Phys. Rev. Lett. 100 (2008) 113901.

[22] A. Alu, N. Engheta, New J. Phys. 10 (2008) 115036.

[23] M.K. Kärkkäinen, Phys. Rev. E 68 (2003) 026602.

[24] G.P. Karman, J.P. Woerdman, Opt. Lett. 23 (1998) 1909.

[25] G.I. Zaginaylov, A. Grudiev, K. Schuenemann, P.V. Turbin, Phys. Rev. Lett. 88 (2002) 195005.

[26] A.E. Serebryannikov, E. Ozbay, Opt. Express 17 (2009) 16869. 